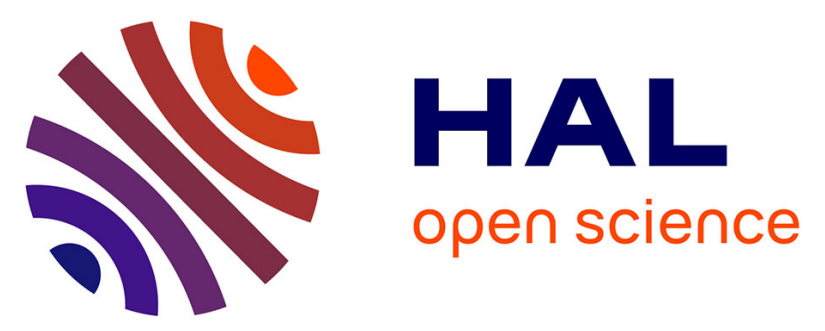

\title{
Synthesis and characterization of half-sandwich ruthenium complexes containing aromatic sulfonamides bearing pyridinyl rings: catalysts for transfer hydrogenation of acetophenone derivatives
}

S. Dayan, N. Ozpozan Kalaycioglu, Jean-Claude Daran, A. Labande, Rinaldo Poli

\section{To cite this version:}

S. Dayan, N. Ozpozan Kalaycioglu, Jean-Claude Daran, A. Labande, Rinaldo Poli. Synthesis and characterization of half-sandwich ruthenium complexes containing aromatic sulfonamides bearing pyridinyl rings: catalysts for transfer hydrogenation of acetophenone derivatives. European Journal of Inorganic Chemistry, 2013, 2013 (18), pp.3224-3232. 10.1002/ejic.201300266 . hal-00993784

\section{HAL Id: hal-00993784 \\ https://hal.science/hal-00993784}

Submitted on 29 Jul 2021

HAL is a multi-disciplinary open access archive for the deposit and dissemination of scientific research documents, whether they are published or not. The documents may come from teaching and research institutions in France or abroad, or from public or private research centers.
L'archive ouverte pluridisciplinaire HAL, est destinée au dépôt et à la diffusion de documents scientifiques de niveau recherche, publiés ou non, émanant des établissements d'enseignement et de recherche français ou étrangers, des laboratoires publics ou privés. 


\title{
Synthesis and characterization of half-sandwich ruthenium complexes containing aromatic sulfonamides bearing pyridinyl rings: catalysts for transfer hydrogenation of acetophenone derivatives
}

\author{
Serkan Dayan, ${ }^{[a]}$ Nilgun Ozpozan Kalaycioglu, $\left.{ }^{[a]}\right]^{*}$, Jean-Claude Daran, ${ }^{[b]}{ }^{[c]}$ Agnès \\ Labande, ${ }^{[b]}$ Rinaldo Poli, ${ }^{[b][c][d]}$
}

\author{
[a] Department of Chemistry, Faculty of Science, Erciyes University, 38039 Kayseri, Turkey \\ Fax: +90-352-437-4933 \\ E-mail: nozpozan@erciyes.edu.tr \\ Homepage: http://aves.erciyes.edu.tr/SorguKimlikBilgileri/aspx?Sorgu=789 \\ [b] CNRS, LCC, 31077 Toulouse, France \\ [c] Univ. Toulouse, UPS, INPT, 31077 Toulouse, France \\ [d] Inst. Univ. France, 75005 Paris, France
}

Keywords: Transfer Hydrogenation / Sulfonamides / Ruthenium / N Ligands

\begin{abstract}
N-quinoline-8-yl-Aryl-benzenesulfonamides (1-6) were successfully synthesized by the reaction of 8 aminoquinoline and various benzenesulfonylchlorides. Then, a series of half-sandwich ruthenium complexes (7-12) were prepared from the reaction of (1-6) with $\left[\mathrm{RuCl}_{2}(\mathrm{p}-\mathrm{cymene})\right]_{2}$. The synthesized compounds were characterized by NMR, FT-IR, EA and X-ray diffraction for compounds 8 and 9. The complexes were screened for their efficiency as catalysts in the transfer hydrogenation (TH) of acetophenone derivatives to phenylethanols in the presence of $\mathrm{KOH}$ with 2-propanol (as hydrogen source) at $82{ }^{\circ} \mathrm{C}$, and show good activity. Complexes $\mathbf{1 0}$ and $\mathbf{1 2}$ were the most active (turnover frequency values: $\mathbf{7 0 3}$ and $\mathbf{7 3 4} \mathrm{h}^{-1}$, respectively).
\end{abstract}




\section{Introduction}

Sulfonamides are widely used in the field of coordination chemistry [1-4] and have also been investigated for their luminescent and antimicrobial properties and for analytical applications [5-7]. Many sulfonamide derivatives and their complexes have been recently reported $\left[8^{\mathrm{a}-\mathrm{d}}\right]$. Furthermore, complexes containing sulfonamide groups have been used to catalyze various organic reactions, for example olefin metathesis, asymmetric transfer hydrogenation, the nitroaldol (Henry) reaction etc. [911]. Sulfonamides containing 8 -aminoquinoline have rarely been studied by the scientific community, mostly in relation to their luminescent properties [12-16]. Also, catalysis and molecular structural properties of sulfonamides have recently been studied in detail by our research team $\left[17^{\mathrm{a}-\mathrm{d}}\right]$.

The ketone transfer hydrogenation $(\mathrm{TH})$ catalyzed by $\mathrm{Ru}(\mathrm{II})$ complexes containing $\mathrm{N}$-donors ligands has attracted increasing attention [18-32] since the success of Noyori's work using 1,2-diamine ligands [33]. Several diamine ligand modifications have been explored aimed at developing catalysts with increased activity. At the same time, half-sandwich ruthenium(II) complexes containing thioamides, 2-(diphenylphosphanyl)-aniline, ferrocenyl tosylamine-phosphine and pyridine-based chelating diamine ligands were also studied for the catalytic transfer hydrogenation of ketones [34-37]. Also, remarkable studies have been carried out in the TH of acetophenone derivatives [38-52].

Herein, a series of new half-sandwich Ru(II) complexes containing sulfonamide ligands were synthesized and characterized by various spectroscopic techniques. The synthesized complexes were used as catalysts for transfer hydrogenation of acetophenone derivatives.

\section{Results and Discussion}

\section{Syntheses}

The synthesis and reaction routes to the ligands and to their corresponding $\mathrm{Ru}(\mathrm{II})$ complexes are presented in Figures 1 and 2. The ligands 1-6 were obtained by the reaction of 8-aminoquinoline with R-arylsulfonyl chlorides in the presence of triethylamine in THF (Figure 1). Then, the new ruthenium complexes (7-12) were synthesized by the reaction of $\mathbf{1 - 6}$ with $\left[\mathrm{RuCl}_{2}(p \text {-cymene })\right]_{2}$ in methyl alcohol (Figure 2). All compounds were characterized by 1H-NMR, 13C-NMR and IR spectroscopy techniques. In addition, the solid state structures of complexes $\mathbf{8}$ and $\mathbf{9}$ were confirmed by X-ray crystallography. 


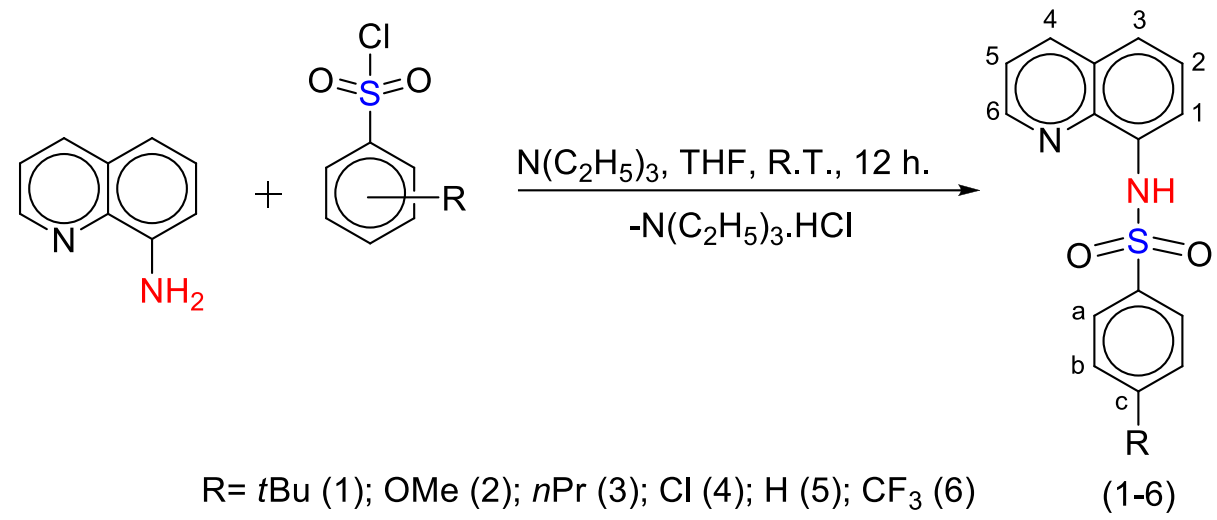

Figure 1. Synthesis of the ligands together with NMR numbering scheme

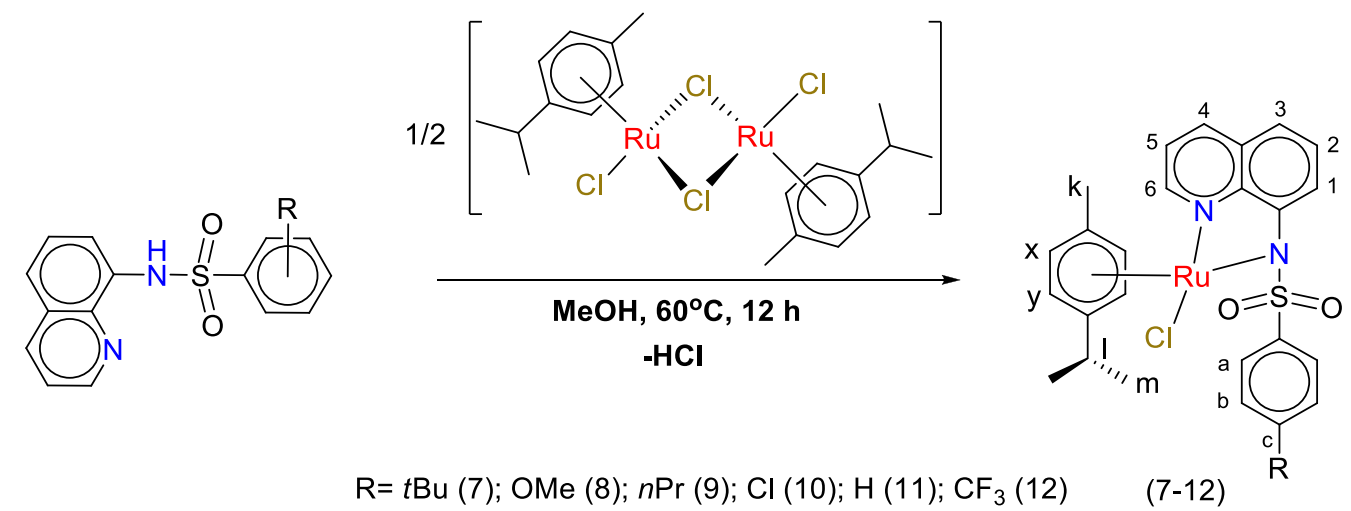

Figure 2. Synthesis of the complexes together with NMR numbering scheme

\section{Crystal Structures}

Molecular views of compounds $\mathbf{8}$ and $\mathbf{9}$ are shown in Figure 3. In each complex the Ru atom has a pseudo-tetrahedral geometry with the $\eta^{6} \pi$-bonded arene rings occupying one vertex of the tetrahedron, the three other vertices being occupied by the two $\mathrm{N}$ atoms of the pyridinyl ligands and the $\mathrm{Cl}$ atoms. Selected bond lengths and angles are shown in Table 1. Such geometry is common for all $\eta^{6}$-ruthenium arene complexes [56-58].
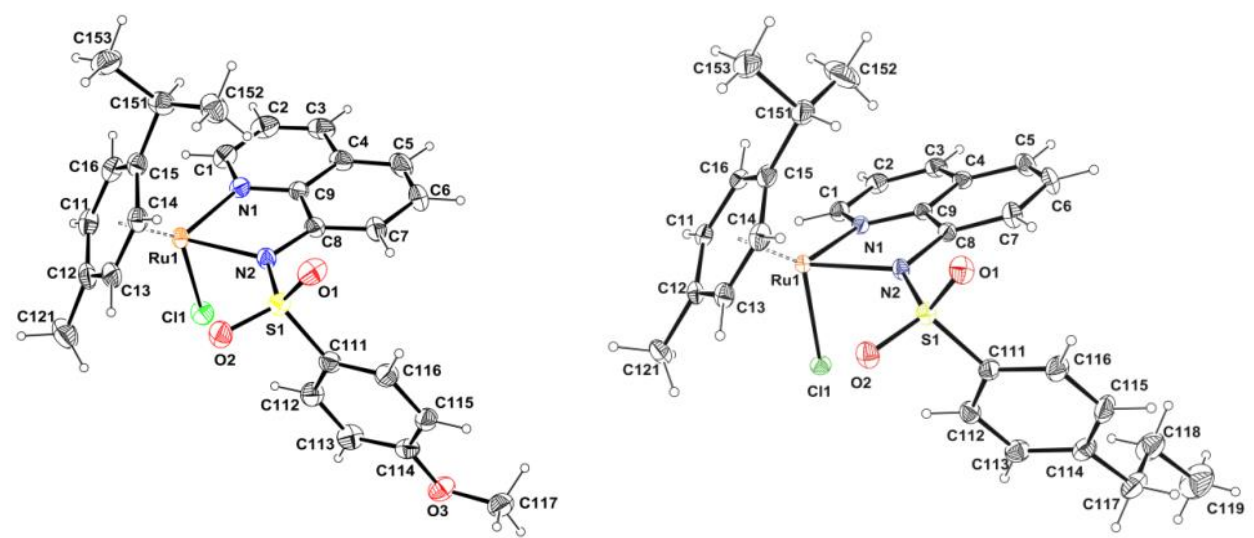

Figure 3: Molecular views of compounds 8 and 9 with the atoms labelling scheme. Ellipsoids are drawn at the $50 \%$ probability level. $\mathrm{H}$ atoms are represented as small spheres of arbitrary radii. 
Table 1: Comparison of selected bond distances $(\AA)$ and bond angles $\left(^{\circ}\right)$ for both compounds 8 and 9. Esds in parentheses refer to the last significant digit.

\begin{tabular}{ll|ll}
\hline \multicolumn{3}{c}{ Compound 8 } & \multicolumn{3}{c}{ Compound 9 } \\
\hline $\mathrm{Ru}(1)-\mathrm{Cg}(1)$ & $1.6799(9)$ & $\mathrm{Ru}(1)-\mathrm{Cg}(1)$ & $1.6666(7)$ \\
$\mathrm{Ru}(1)-\mathrm{N}(1)$ & $2.0965(16)$ & $\mathrm{Ru}(1)-\mathrm{N}(1)$ & $2.0852(14)$ \\
$\mathrm{Ru}(1)-\mathrm{N}(2)$ & $2.1285(16)$ & $\mathrm{Ru}(1)-\mathrm{N}(2)$ & $2.1236(13)$ \\
$\mathrm{Ru}(1)-\mathrm{Cl}(1)$ & $2.4322(5)$ & $\mathrm{Ru}(1)-\mathrm{Cl}(1)$ & $2.3981(5)$ \\
$\mathrm{S}(1)-\mathrm{O}(2)$ & $1.4382(15)$ & $\mathrm{S}(1)-\mathrm{O}(2)$ & $1.4392(13)$ \\
$\mathrm{S}(1)-\mathrm{O}(1)$ & $1.4424(16)$ & $\mathrm{S}(1)-\mathrm{O}(1)$ & $1.4496(12)$ \\
$\mathrm{S}(1)-\mathrm{N}(2)$ & $1.6157(16)$ & $\mathrm{S}(1)-\mathrm{N}(2)$ & $1.6146(14)$ \\
$\mathrm{S}(1)-\mathrm{C}(111)$ & $1.768(2)$ & $\mathrm{S}(1)-\mathrm{C}(111)$ & $1.7774(17)$ \\
$\mathrm{N}(1)-\mathrm{C}(1)$ & $1.324(3)$ & $\mathrm{N}(1)-\mathrm{C}(1)$ & $1.330(2)$ \\
$\mathrm{N}(1)-\mathrm{C}(9)$ & $1.373(3)$ & $\mathrm{N}(1)-\mathrm{C}(9)$ & $1.377(2)$ \\
$\mathrm{N}(2)-\mathrm{C}(8)$ & $1.400(2)$ & $\mathrm{N}(2)-\mathrm{C}(8)$ & $1.406(2)$ \\
$\mathrm{Cg}(1)-\mathrm{Ru}(1)-\mathrm{Cl}(1)$ & $127.01(4)$ & $\mathrm{Cg}(1)-\mathrm{Ru}(1)-\mathrm{Cl}(1)$ & $127.27(3)$ \\
$\mathrm{Cg}(1)-\mathrm{Ru}(1)-\mathrm{N}(1)$ & $131.54(5)$ & $\mathrm{Cg}(1)-\mathrm{Ru}(1)-\mathrm{N}(1)$ & $130.32(4)$ \\
$\mathrm{Cg}(1)-\mathrm{Ru}(1)-\mathrm{N}(2)$ & $131.93(5)$ & $\mathrm{Cg}(1)-\mathrm{Ru}(1)-\mathrm{N}(2)$ & $77.10(5)$ \\
$\mathrm{N}(1)-\mathrm{Ru}(1)-\mathrm{N}(2)$ & $76.86(6)$ & $\mathrm{N}(1)-\mathrm{Ru}(1)-\mathrm{N}(2)$ & $85.09(4)$ \\
$\mathrm{N}(1)-\mathrm{Ru}(1)-\mathrm{Cl}(1)$ & $84.31(5)$ & $\mathrm{N}(1)-\mathrm{Ru}(1)-\mathrm{Cl}(1)$ & $116.60(8)$ \\
$\mathrm{N}(2)-\mathrm{Ru}(1)-\mathrm{Cl}(1)$ & $87.46(5)$ & $\mathrm{N}(2)-\mathrm{Ru}(1)-\mathrm{Cl}(1)$ & $107.34(7)$ \\
$\mathrm{O}(2)-\mathrm{S}(1)-\mathrm{O}(1)$ & $116.55(10)$ & $\mathrm{O}(2)-\mathrm{S}(1)-\mathrm{O}(1)$ & $112.02(7)$ \\
$\mathrm{O}(2)-\mathrm{S}(1)-\mathrm{N}(2)$ & $107.43(9)$ & $\mathrm{O}(2)-\mathrm{S}(1)-\mathrm{N}(2)$ & $108.86(8)$ \\
$\mathrm{O}(1)-\mathrm{S}(1)-\mathrm{N}(2)$ & $111.55(9)$ & $\mathrm{O}(1)-\mathrm{S}(1)-\mathrm{N}(2)$ & $106.36(8)$ \\
$\mathrm{O}(2)-\mathrm{S}(1)-\mathrm{C}(111)$ & $107.18(10)$ & $\mathrm{O}(2)-\mathrm{S}(1)-\mathrm{C}(111)$ & $105.03(7)$ \\
$\mathrm{O}(1)-\mathrm{S}(1)-\mathrm{C}(111)$ & $106.93(9)$ & $\mathrm{O}(1)-\mathrm{S}(1)-\mathrm{C}(111)$ & $119.04(15)$ \\
$\mathrm{N}(2)-\mathrm{S}(1)-\mathrm{C}(111)$ & $106.70(9)$ & $\mathrm{N}(2)-\mathrm{S}(1)-\mathrm{C}(111)$ & $125.18(11)$ \\
$\mathrm{C}(1)-\mathrm{N}(1)-\mathrm{C}(9)$ & $118.80(17)$ & $\mathrm{C}(1)-\mathrm{N}(1)-\mathrm{C}(9)$ & $115.69(11)$ \\
$\mathrm{C}(1)-\mathrm{N}(1)-\mathrm{Ru}(1)$ & $125.07(14)$ & $\mathrm{C}(1)-\mathrm{N}(1)-\mathrm{Ru}(1)$ & $119.50(11)$ \\
$\mathrm{C}(9)-\mathrm{N}(1)-\mathrm{Ru}(1)$ & $116.10(12)$ & $\mathrm{C}(9)-\mathrm{N}(1)-\mathrm{Ru}(1)$ & $114.30(10)$ \\
$\mathrm{C}(8)-\mathrm{N}(2)-\mathrm{S}(1)$ & $118.75(13)$ & $\mathrm{C}(8)-\mathrm{N}(2)-\mathrm{S}(1)$ & $122.31(16)$ \\
$\mathrm{C}(8)-\mathrm{N}(2)-\mathrm{Ru}(1)$ & $115.35(12)$ & $\mathrm{C}(8)-\mathrm{N}(2)-\mathrm{Ru}(1)$ & \\
$\mathrm{S}(1)-\mathrm{N}(2)-\mathrm{Ru}(1)$ & $125.75(9)$ & $\mathrm{S}(1)-\mathrm{N}(2)-\mathrm{Ru}(1)$ & $\mathrm{N}(1)-\mathrm{C}(1)-\mathrm{C}(2)$ \\
$\mathrm{N}(1)-\mathrm{C}(1)-\mathrm{C}(2)$ & $122.4(2)$ & & \\
\hline & & & \\
\hline
\end{tabular}

\section{NMR Spectra}

In the ${ }^{1} \mathrm{H}-\mathrm{NMR}$ spectra of the ligands $\mathbf{1 - 6}$, the $-H_{a},-H_{b}$ and $-H_{c}$ protons were observed as a doublet and two triplets, respectively, in a 2:2:1 ratio at around $\delta 7.79-7.88 \mathrm{ppm}$ in $N$-quinoline-8-ylbenzenesulfonamide. Similarly in other $N$-quinoline-8-yl-arylsulfonamides ligands, the $-H_{a}$ and $-H_{b}$ protons were observed as doublets in a 2:2 ratio at around $\delta 6.99-8.80 \mathrm{ppm}$. The $N H$ protons were found at around $\delta 9.31-11.66 \mathrm{ppm}$.

In the ${ }^{1} \mathrm{H}-\mathrm{NMR}$ spectra for $\mathbf{7 - 1 2}$, the sulfonamide $\mathrm{NH}$ proton is no longer present while new resonances belonging to the $p$-cymene group. $\left(-H_{k},-H_{x},-H_{y},-H_{l}\right.$ and $\left.-H_{m}\right)$ appear shown in Table 2. 
Table 2. ${ }^{1} \mathrm{H}-\mathrm{NMR}$ and ${ }^{13} \mathrm{C}-\mathrm{NMR}$ data of ligands and complexes

\begin{tabular}{|c|c|c|c|c|c|c|c|c|c|c|}
\hline \multirow[t]{2}{*}{ Ligands } & \multicolumn{10}{|c|}{${ }^{1} \mathrm{H}-\mathrm{NMR} /{ }^{13} \mathrm{C}-\mathrm{NMR}$ (ppm) } \\
\hline & $-H_{a}$ & \multicolumn{2}{|c|}{$-H_{b}$} & $-H_{c}$ & $\mathrm{NH}$ & \multicolumn{2}{|c|}{$-p-\mathrm{C}\left(\mathrm{CH}_{3}\right)_{3}$} & \multicolumn{2}{|r|}{$-p-\mathrm{OCH}_{3}$} & $-p-\mathrm{CH}_{2} \mathrm{CH}_{2} \mathrm{CH}_{3}$ \\
\hline \multirow[t]{2}{*}{1} & \multirow{2}{*}{\multicolumn{3}{|c|}{7.86}} & \multirow[t]{2}{*}{-} & \multirow[t]{2}{*}{9.31} & & $1.24\left({ }^{1} \mathrm{H}\right)$ & \multirow{2}{*}{\multicolumn{2}{|c|}{-}} & \multirow[t]{2}{*}{-} \\
\hline & & & & & & \multicolumn{2}{|c|}{$35.0,30.9\left({ }^{13} \mathrm{C}\right)$} & & & \\
\hline 2 & 7.80 & 6.9 & & - & 10.02 & & - & 3.74 & $\left.\left({ }^{1} \mathrm{H}\right) / 56.1-{ }^{13} \mathrm{C}\right)$ & - \\
\hline \multirow[t]{2}{*}{3} & \multirow[t]{2}{*}{8.80} & \multirow{2}{*}{\multicolumn{2}{|c|}{8.20}} & \multirow[t]{2}{*}{-} & \multirow[t]{2}{*}{11.66} & \multirow{2}{*}{\multicolumn{2}{|c|}{-}} & \multirow{2}{*}{\multicolumn{2}{|c|}{ - }} & $0.84,3.12,1.40\left({ }^{1} \mathrm{H}\right)$ \\
\hline & & & & & & & & & & $45.9,24.0,8.7\left({ }^{13} \mathrm{C}\right)$ \\
\hline 4 & 7.83 & \multicolumn{2}{|c|}{7.80} & - & 11.00 & & - & \multicolumn{2}{|r|}{-} & - \\
\hline 5 & 7.86 & \multicolumn{2}{|c|}{7.79} & 7.88 & 11.21 & & - & \multicolumn{2}{|r|}{-} & - \\
\hline 6 & 8.03 & \multicolumn{2}{|c|}{\begin{tabular}{|l|l|}
7.86 & \\
\end{tabular}} & - & 10.94 & & - & \multicolumn{2}{|r|}{-} & - \\
\hline Complexes & $-H_{k}$ & $-H_{x}$ & $-H_{y}$ & $-H_{l}$ & $-H$ & & $-p-\mathrm{C}(C$ & $\frac{\left.H_{3}\right)_{3}}{2}$ & $-p-\mathrm{OCH}_{3}$ & $-p-\mathrm{CH}_{2} \mathrm{CH}_{2} \mathrm{CH}_{3}$ \\
\hline \multirow[t]{2}{*}{7} & 2.37 & \multirow[t]{2}{*}{6.29} & \multirow[t]{2}{*}{5.70} & \multirow[t]{2}{*}{2.62} & \multirow{2}{*}{\multicolumn{2}{|c|}{$0.95-1.07$}} & 1.24( & & - & - \\
\hline & & & & & & & $34.8,31$. & $\left({ }^{13} \mathrm{C}\right)$ & & \\
\hline 8 & 2.36 & 6.26 & 5.67 & 2.62 & 0.92 & .08 & - & & $\begin{array}{l}3.72\left({ }^{1} \mathrm{H}\right) / \\
55.3\left({ }^{13} \mathrm{C}\right)\end{array}$ & - \\
\hline 9 & 2.34 & 6.24 & 5.86 & 2.61 & 0.92 & .07 & - & & - & $0.83,3.10,1.37\left({ }^{1} \mathrm{H}\right)$ \\
\hline & & & & & & & & & & $46.1,24.1,8.7\left({ }^{13} \mathrm{C}\right)$ \\
\hline 10 & 2.36 & 6.23 & 5.67 & 2.63 & 0.94 & .09 & - & & - & - \\
\hline 11 & 2.37 & 6.26 & 5.69 & 2.62 & 0.93 & .08 & - & & - & - \\
\hline 12 & 2.36 & 6.23 & 5.68 & 2.63 & 0.94 & 1.09 & - & & - & - \\
\hline
\end{tabular}

\section{Infrared Spectra}

The sulfonamide $N-H$ stretching band for the ligands 1-6 appears at $3260-3192 \mathrm{~cm}^{-1}$ see Table 3 . This band disappears on going to the ruthenium complexes 7-12. The frequencies of the $\mathrm{SO}_{2}$ stretching and bending peaks are shown in Table 3. For 7-12, there is a blue shift for the bending and asymmetric stretching are observed a general shift toward increase frequency as compared to their respective ligands. But, the symmetric stretching was also seen a general shift toward lower frequency as compared to their respective ligands.

Table 3. IR data of ligands and complexes

\begin{tabular}{|c|c|c|c|c|}
\hline \multirow[t]{2}{*}{ Ligand } & \multicolumn{4}{|c|}{ IR $\left(\mathrm{cm}^{-1}\right)$} \\
\hline & $v(\mathrm{NH})$ & $\mathrm{v}_{\mathrm{as}}\left(\mathrm{SO}_{2}\right)^{\mathrm{a}}$ & $v_{s}\left(\mathrm{SO}_{2}\right)^{b}$ & $\Delta\left(\mathbf{S O}_{2}\right)^{c}$ \\
\hline 1 & 3258 & 1303 & 1162 & 570 \\
\hline 2 & 3192 & 1332 & 1153 & 572 \\
\hline 3 & 3260 & 1306 & 1156 & 560 \\
\hline 4 & 3243 & 1303 & 1157 & 555 \\
\hline 5 & 3217 & 1307 & 1169 & 557 \\
\hline 6 & 3239 & 1307 & 1163 & 551 \\
\hline Complex & $v_{\text {as }}\left(\mathrm{SO}_{2}\right)$ & & & $\Delta\left(\mathbf{S O}_{2}\right)$ \\
\hline 7 & 1315 & & & 577 \\
\hline 8 & 1316 & & & 572 \\
\hline 9 & 1316 & & & 577 \\
\hline 10 & 1315 & & & 567 \\
\hline 11 & 1316 & & & 582 \\
\hline 12 & 1317 & & & 563 \\
\hline
\end{tabular}

${ }^{a} v_{\text {as }}=$ asymmetric stretching. ${ }^{b} v_{\mathrm{s}}=$ symmetric stretching. ${ }^{\mathrm{c}} \Delta=$ bending 


\section{Catalytic Studies}

As the starting point, the performances of the catalysts in the transfer hydrogenation were screened by using acetophenone as a model substrate and we screened the influence of the base. $\mathrm{NaOH}$ and $\mathrm{KOH}$ are known to yield better conversions when compared to $\mathrm{K}_{2} \mathrm{CO}_{3}$ and $\mathrm{KOBu}^{\mathrm{t}}$ in the $\mathrm{TH}$ reactions [36, 49]. The stronger the base the higher the general conversion rankings, $\mathrm{KOH}>\mathrm{NaOH}>\mathrm{KOBu}^{\mathrm{t}}>\mathrm{K}_{2} \mathrm{CO}_{3}$. Indeed, the $\mathrm{TH}$ of acetophenone catalyzed by compound $\mathbf{1 0}$ leads to better results when using $\mathrm{KOH}$, reaching $97 \%$ conversion within $120 \mathrm{~min}$ at a substrate/catalyst/base $(\mathrm{S} / \mathrm{C} / \mathrm{base})$ molar ratio of 1: 0.002 : 10 (Figure 4). In the absence of a base no transfer hydrogenation was observed. Therefore, KOH was selected as the base in all subsequent studies.

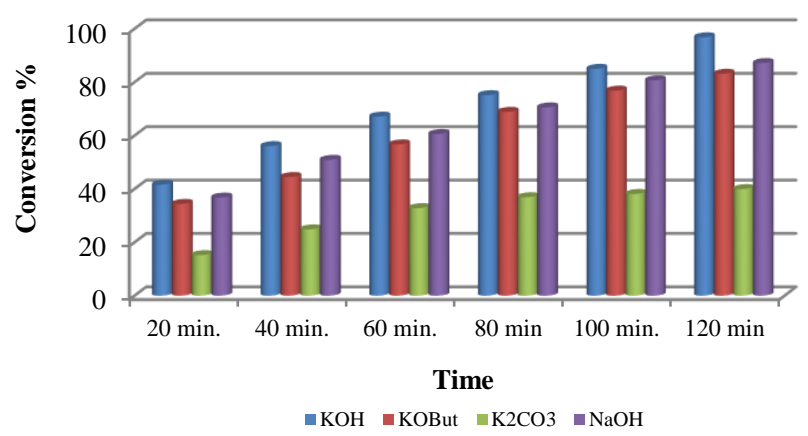

Figure 4. Effect of various bases in the TH of acetophenone catalyzed by $\mathbf{1 0}(0.2 \mathrm{~mol} \%)$ in 2-propanol at $82{ }^{\circ} \mathrm{C}$.

A variety of ketones was transformed to the corresponding secondary alcohols. Typical results are shown in Figure 5. The transformation of 4-chloroacetophenone is the fastest, giving complete conversion to the corresponding alcohol 120 min under the selected operating conditions (Figure 5, b). Among the tested catalysts, complexes 8, 10 and 12 are more efficient than complexes 7, 9 and $\mathbf{1 1}$. 

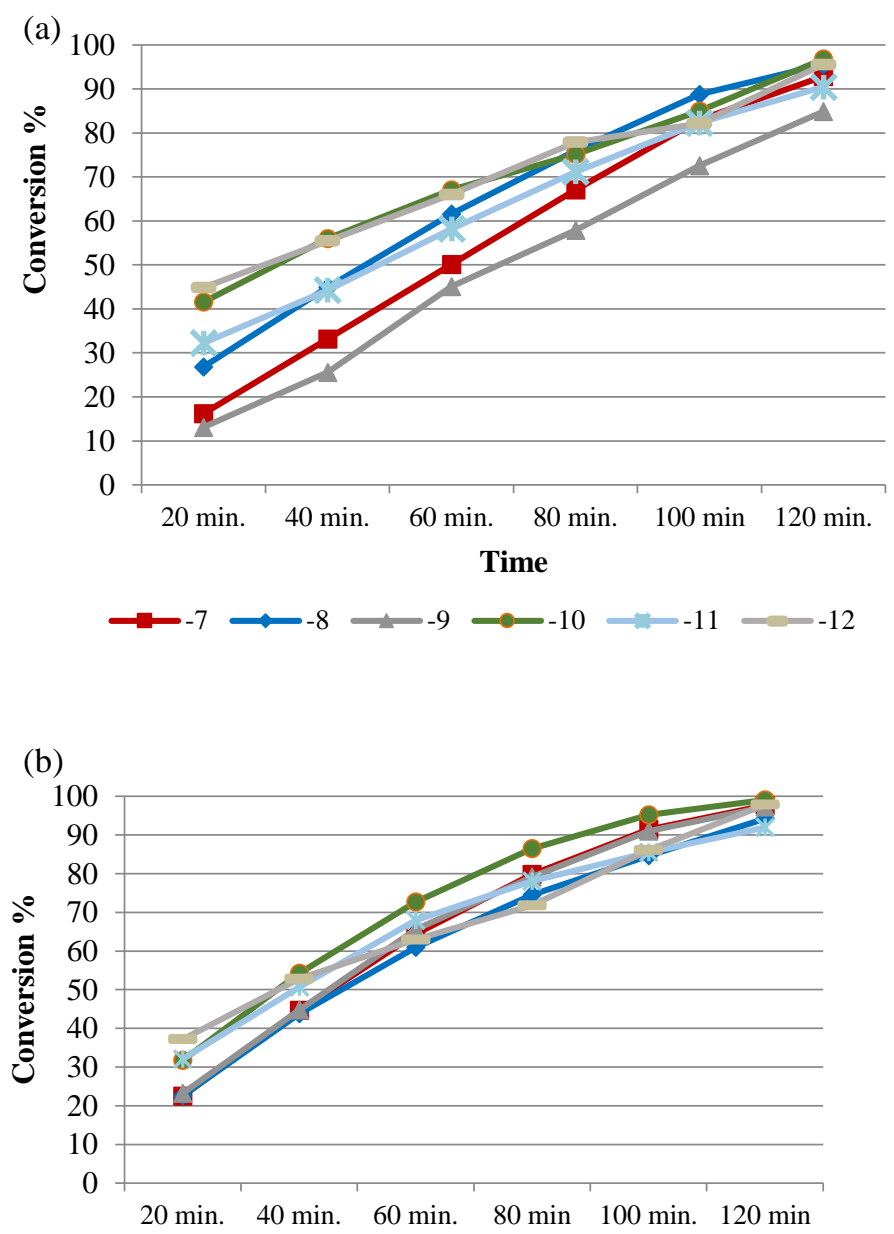

Time

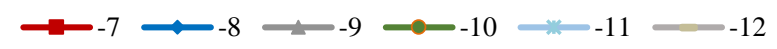

(c)

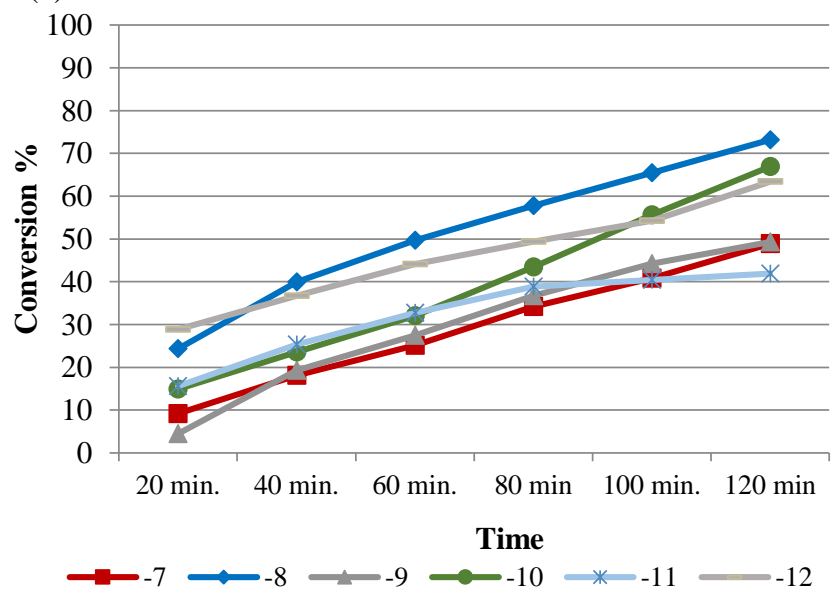

Figure 5. Catalytic activity as shown by the $\%$ conversion vs. time for the transfer hydrogenation of (a) acetophenone, (b) 4-chloro-acetophenone, (c) 4-methyl-acetophenone catalyzed by compounds 712 in isopropanol. Conditions: ketone/Ru/KOH, 1:0.002:10; $\mathrm{T}=82{ }^{\circ} \mathrm{C}$ 
We have also examined the acetophenone $\mathrm{TH}$ at a (S/C/base) molar ratio of 1: 0.001: 10 (Figure 6). The results of the catalytic experiments show once again that complexes $\mathbf{1 0}$ and $\mathbf{1 2}$ are highly efficient. The highest measured TOF were in the $734-575 \mathrm{~h}^{-1}$ range for the S/C molar ratio of 1: 0.001, at $20 \mathrm{~min}$. (Table 4).

Table-5 Initial TOF for the TH of various ketones catalyzed by compounds 7-12.

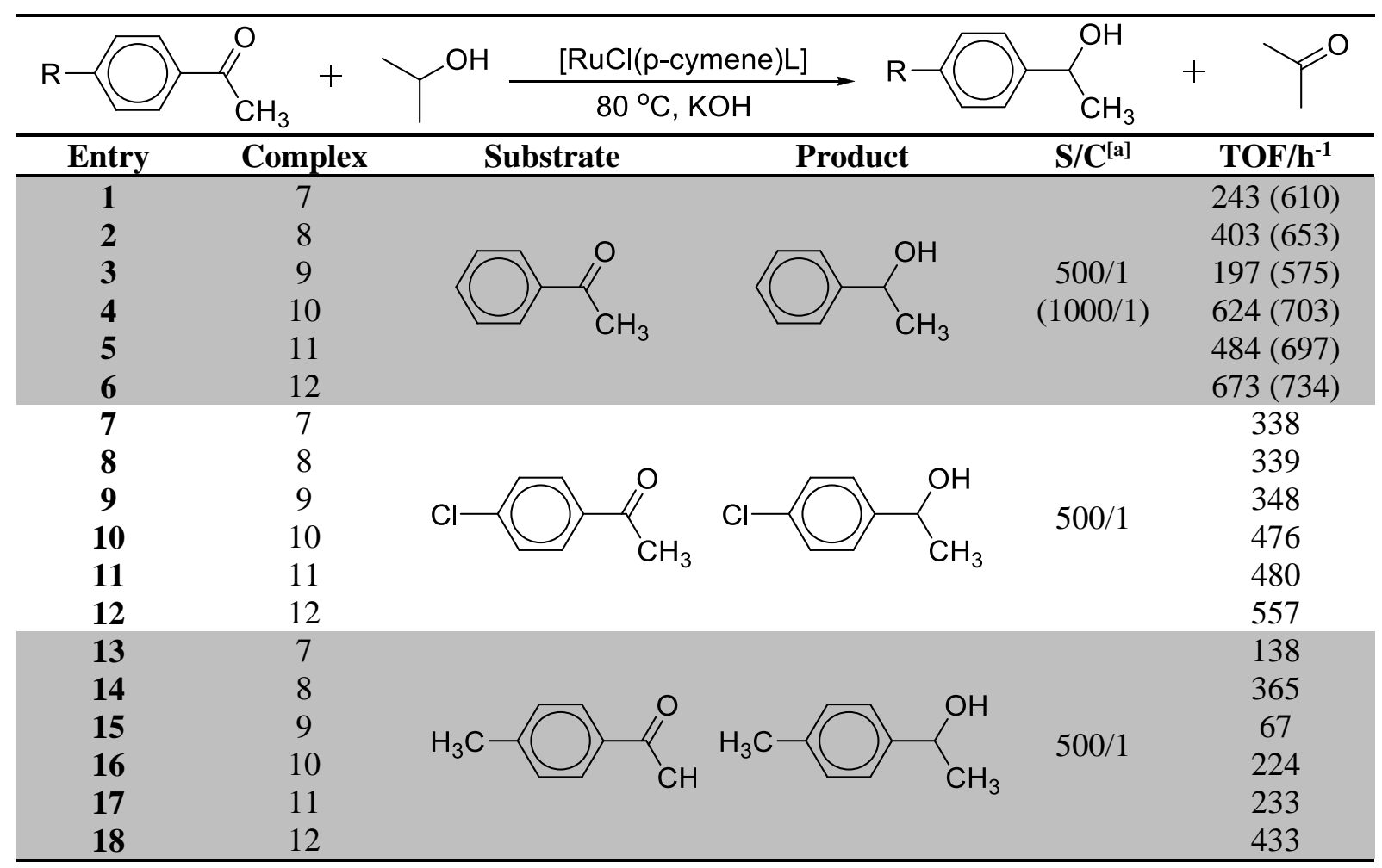

[a] Acetophenone/Ru molar ratio.

\section{Conclusions}

In summary, we have reported the preparation and characterization of the sulfonamide ligands 1-6, and their neutral half-sandwich sulfonamido-Ru(II) complexes 7-12. We have also reported the catalytic activity of these complexes in the transfer hydrogenation of some acetophenone derivatives with the use of 2-propanol in the presence of base. Complexes $\mathbf{1 0}$ and $\mathbf{1 2}$ are the most active complexes. The procedure is simple and efficient towards various aryl ketones. The catalyst efficiency depends not only on the ligand but also on the aromatic ketone substituent. Electron-withdrawing groups introduced at the para position of the acetophenone accelerate the transformation, whereas electron-donating groups slow it down. Likewise, the presence of electron-withdrawing groups on the sulfonamido-ring has a beneficial effect. The catalytic activity decreases in the order $10>12>8>7>9>11$, the best results being obtained in the presence of the $-\mathrm{Cl}$ and $-\mathrm{CF}_{3}$ substituents at para position of the sulfonamido aryl 
group. Ruthenium(II) arene complexes widely used in the TH of ketones. When examined to the literature, it is clear that the half-sandwich sulfonamido- $\mathrm{Ru}$ (II) complexes used herein have been a good efficiency in the TH [59-73].

\section{Experimental Section}

Materials and Methods. All reagents and solvents were obtained from commercial suppliers and used without any additional purification. NMR spectra were recorded in $5 \mathrm{~mm}$ tubes at $297 \mathrm{~K}$ on a Bruker Avance III $400 \mathrm{NMR}$ spectrometer at $400 \mathrm{MHz}\left({ }^{1} \mathrm{H}\right)$ and $100.56 \mathrm{MHz}\left({ }^{13} \mathrm{C}\right)$. Signals are quoted in parts per million as $\delta$ downfield from tetramethylsilane $(\delta 0.00)$ as an internal standard. Coupling constants ( $J$-values) are given in hertz. Abbreviations used for the NMR resonances are: br= broad, $\mathrm{s}=$ singlet, $\mathrm{d}$ $=$ doublet, $\mathrm{t}=$ triplet, $\mathrm{m}=$ multiplet. Infrared spectra were measured with a Perkin-Elmer Spectrum 400 FTIR system and recorded using a universal ATR sampling accessory within the range 550-4000 $\mathrm{cm}^{-1}$. Melting points were determined in open capillary tubes on a digital Electrothermal 9100 melting point apparatus. GC measurements for catalytic experiments were performed using Fisons 8000 Series instrument equipped with a DB-1MS column $(30 \mathrm{mx} 0.32 \mathrm{mmx} 0.25 \mathrm{~m}) \&$ Younglin Acme 6100, OPTIMA 5 MS column (30mx0.32mmx0.25m).

$\mathrm{TOF}=$ moles of product $/$ (moles of the catalyst) $\mathrm{x}$ (hour), measured on the basis of the conversion at 20 min for entries 1-18, at 30 min for entries 19-24. Conditions are given in the legends of Fig. 5 and 6.

\section{General procedure for the synthesis of Ligands (L), 1-6}

$\mathrm{N}$-quinoline-8-yl-aryl-benzenesulfonamides (1-6) were prepared by modifying the published procedure [44]. A $50 \mathrm{ml}$ Schlenk tube containing a magnetic stirring bar was charged with a solution of the suitable benzenesulfonylchloride derivative $(10 \mathrm{mmol})$ in THF $(10 \mathrm{ml})$. The slow addition of a solution of triethylamine (20 mmol) in THF (5 ml) and 8-aminoquinoline $(10 \mathrm{mmol})$ in THF (5 ml), respectively, gives rise to the immediate precipitation of a white solid $\left(\mathrm{HCl} \cdot \mathrm{N}\left(\mathrm{C}_{2} \mathrm{H}_{5}\right)_{3}\right)$. After being stirred for 12 hours at room temperature, the solid was removed by filtration using a fine sintered-glass filter. Then, the solvent was completely removed under reduced pressure. The crude product was used without further purification. An analytically pure sample was obtained by recrystallization from chloroform/diethyl ether (15 ml 1:3, v/v) (Fig. 1).

N- Quinoline-8-yl -4-t-butyl-benzenesulfonamide (1): Yield: $85 \%$. Orange solid, Mp: $155-157^{\circ} \mathrm{C}$, ${ }^{1} \mathrm{H}-\mathrm{NMR}\left(\mathrm{CDCl}_{3}, \delta \mathrm{ppm}\right): 1.24$ (s, 9H, -C(CH$)_{3}, 7.37-7.48\left(\mathrm{~m}, 4 \mathrm{H},-\mathrm{H}_{2-5}\right), 7.86$ (dd, 4H, $J=4 \mathrm{~Hz}, J=4$ $\left.\mathrm{Hz},-H_{a-b}\right), 8.13\left(\mathrm{~d}, 1 \mathrm{H}, J=8 \mathrm{~Hz},-H_{l}\right), 8.77\left(\mathrm{~d}, 1 \mathrm{H}, J=8 \mathrm{~Hz},-H_{6}\right), 9.31$ (br. $\left.1 \mathrm{H},-N H\right) .{ }^{13} \mathrm{C}-\mathrm{NMR}\left(\mathrm{CDCl}_{3}\right.$, ppm): 30.9 (-C(CH3) $\left.)_{3}\right), 35.0\left(-C\left(\mathrm{CH}_{3}\right)_{3}\right), 115.1$ (Ar. -C), 121.9 (Ar. -C), 122.0 (Ar. -C), 125.9 (Ar. -C), 127.0 (Ar. -C), 128.2 (Ar. -C), 133.8 (Ar. -C), 136.4 (Ar. -C), 136.6 (Ar. -C), 138.2 (Ar. -C), 148.4 (Ar. -C), 148.5 (Ar. -C), 156.7 (Ar. -C). IR ( $\left.\mathrm{cm}^{-1}\right)$ : 3258 (-NH), 3078, 2962, 2905, 2869, 1623, 1595, 1579, 1505, 1471, 1436, 1414, 1397, 1378, 1362, 1336, $1303\left(-\mathrm{SO}_{2}\right), 1266,1236,1206,1194,1162\left(-\mathrm{SO}_{2}\right)$, 
1139, 1112, 1086, 1057, 1029, 1013, 984, 972, 962, 922, 894, 851, 824, 803, 792, 749, 734, 645, 637, 626, 600, $570\left(-\mathrm{SO}_{2}\right), 548,515,478,466 . \mathrm{C}_{19} \mathrm{H}_{20} \mathrm{~N}_{2} \mathrm{O}_{2} \mathrm{~S}$ (340.3): calcd. for: C:67.03, H:5.92, N:8.23, O:9.40, S:9.42, Found: C:67.15, H:5.84, N:8.18, S:9.47.

N-Quinoline-8-yl-4-methoxy-benzenesulfonamide (2): Yield: $78 \%$, brown solid, Mp: $145-147{ }^{\circ} \mathrm{C}$, ${ }^{1} \mathrm{H}-\mathrm{NMR}$ (DMSO, $\delta \mathrm{ppm}$ ): 3.74 (s, 3H, -OCH $H_{3}, 6.99$ (d, 2H, $J=8 \mathrm{~Hz},-H_{b}$ ), 7.55-7.77 (m, 4H, - $H_{2-5}$ ), $7.80\left(\mathrm{~d}, 2 \mathrm{H}, J=8 \mathrm{~Hz},-H_{a}\right), 8.55$ (d, $\left.1 \mathrm{H}, J=8 \mathrm{~Hz},-H_{l}\right), 8.95$ (d, 1H, J=8 Hz, - $\left.H_{6}\right), 10.02$ (br. $\left.1 \mathrm{H},-N H\right)$. ${ }^{13} \mathrm{C}-\mathrm{NMR}$ (DMSO, ppm): 56.1 (-OCH ), 114.8 (Ar. -C), 119.7 (Ar. -C), 122.9 (Ar. -C), 124.3 (Ar. -C), 127.8 (Ar. -C), 128.9 (Ar. -C), 129.8 (Ar. -C), 130.9 (Ar. -C), 132.9 (Ar. -C), 138.0 (Ar. -C), 139.4 (Ar. -C), 149.1 (Ar. -C), 163.1 (Ar. -C). IR ( $\left.\mathrm{cm}^{-1}\right)$ : $3192(-\mathrm{NH}), 3192(-\mathrm{NH}), 3045,2971,2872,2776,2731$, $1629,1590,1576,1543,1489,1465,1436,1425,1408,1375,1347,1332\left(-\mathrm{SO}_{2}\right), 1310,1298,1285$, 1256, 1220, 1206, 1178, 1153 (-SO $\mathrm{SO}_{2}, 1138,1113,1089,1061,1025,1006,974,935,924,909,885$, $849,828,803,779,768,741,698,638,627,605,572\left(-\mathrm{SO}_{2}\right), 555,538,516,500,484,466 . \mathrm{C}_{16} \mathrm{H}_{14} \mathrm{~N}_{2} \mathrm{O}_{3} \mathrm{~S}$ (314.2): Calcd. for: C: 61.13, H: 4.49, N:8.91, O: 15.27, S: 10.20, Found: C: 61.19, H: 4.39, N:8.81, S: 10.11 .

N-Quinoline-8-yl-4-n-propyl-benzenesulfonamide (3): Yield: $82 \%$, light brown solid, Mp: 120-122 ${ }^{\circ} \mathrm{C},{ }^{1} \mathrm{H}-\mathrm{NMR}\left(\mathrm{CDCl}_{3}, \delta \mathrm{ppm}\right): 0.84$ (t, $\left.2 \mathrm{H}, \mathrm{J}=8 \mathrm{~Hz},-\mathrm{CH}_{2} \mathrm{CH}_{2} \mathrm{CH}_{3}\right), 1.40$ (t, $3 \mathrm{H}, J=8 \mathrm{~Hz},-\mathrm{CH}_{2} \mathrm{CH}_{2} \mathrm{CH}_{3}$ ), $3.12\left(\mathrm{~m}, 2 \mathrm{H},-\mathrm{CH}_{2} \mathrm{CH}_{2} \mathrm{CH}_{3}\right), 7.14-7.89\left(\mathrm{~m}, 4 \mathrm{H},-\mathrm{H}_{2-5}\right), 8.20$ (d, 2H, J=8 Hz, $\left.-H_{b}\right), 8.74(\mathrm{~d}, 1 \mathrm{H}, J=8 \mathrm{~Hz},-$ $\left.H_{l}\right), 8.80\left(\mathrm{~d}, 2 \mathrm{H}, J=8 \mathrm{~Hz},-H_{a}\right), 8.96\left(\mathrm{~d}, 1 \mathrm{H}, J=8 \mathrm{~Hz},-H_{6}\right), 11.66$ (br. $\left.1 \mathrm{H},-\mathrm{NH}\right) .{ }^{13} \mathrm{C}-\mathrm{NMR}\left(\mathrm{CDCl}_{3}, \mathrm{ppm}\right)$ : $8.7\left(-\mathrm{CH}_{2} \mathrm{CH}_{2} \mathrm{CH}_{3}\right), 24.0\left(-\mathrm{CH}_{2} \mathrm{CH}_{2} \mathrm{CH}_{3}\right), 45.9\left(-\mathrm{CH}_{2} \mathrm{CH}_{2} \mathrm{CH}_{3}\right), 116.5$ (Ar. -C), 120.3 (Ar. -C), 121.8 (Ar. -C), 122.4 (Ar. -C), 125.9 (Ar. -C), 127.3 (Ar. -C), 128.3 (Ar. -C), 128.9 (Ar. -C), 131.3 (Ar. -C), 133.3 (Ar. -C), 136.6 (Ar. -C), 147.8 (Ar. -C), 148.4 (Ar. -C). IR (cm $\left.{ }^{-1}\right): 3260$ (-NH), 3068, 2959, 2932 , 2866, 2605, 2498, 1662, 1623, 1593, 1543, 1504, 1471, 1446, 1435, 1429, 1408, 1378, 1367, 1330, 1306 $\left(-\mathrm{SO}_{2}\right), 1248,1237,1223,1184,1156\left(-\mathrm{SO}_{2}\right), 1119,1112,1086,1058,1029,1018,999,970,921,883$, $844,829,817,806,795,758,728,690,679,637,601,575,560\left(-\mathrm{SO}_{2}\right), 535,526,485,470 . \mathrm{C}_{18} \mathrm{H}_{18} \mathrm{~N}_{2} \mathrm{O}_{2} \mathrm{~S}$ (326.3): calcd. for: C:66.23, H: 5.56, N: 8.58, O: 9.80, S: 9.82, Found: C: 66.35, H: 5.61, N: 8.49, S: 9.95 .

N-Quinoline-8-yl-4-chloro-benzenesulfonamide (4): Yield: $81 \%$, brown solid, Mp: $110-112{ }^{\circ} \mathrm{C},{ }^{1} \mathrm{H}$ NMR ( $\left.\mathrm{CDCl}_{3}, \delta \mathrm{ppm}\right)$ : 7.30-7.49 (m, 4H, - $\left.H_{2-5}\right), 7.80$ (d, 2H, $\left.J=8 \mathrm{~Hz},-H_{b}\right), 7.83\left(\mathrm{~d}, 2 \mathrm{H}, J=8 \mathrm{~Hz},-H_{a}\right)$, $8.10\left(\mathrm{~d}, 1 \mathrm{H}, J=8 \mathrm{~Hz},-H_{1}\right), 8.74$ (d, $\left.1 \mathrm{H}, J=8 \mathrm{~Hz},-H_{6}\right), 11.00$ (br. $\left.1 \mathrm{H},-N H\right) .{ }^{13} \mathrm{C}-\mathrm{NMR}\left(\mathrm{CDCl}_{3}, \mathrm{ppm}\right)$ : 115.4 (Ar. -C), 116.0 (Ar. -C), 122.1 (Ar. -C), 122.6 (Ar. -C), 126.8 (Ar. -C), 127.6 (Ar. -C), 128.3 (Ar. -C), 128.6 (Ar. -C), 129.2 (Ar. -C), 133.4 (Ar. -C), 136.3 (Ar. -C), 139.4 (Ar. -C), 148.9 (Ar. -C). IR $\left(\mathrm{cm}^{-1}\right): 3243$ (-NH), 3089, 3019, 2978, 2947, 1618, 1581, 1504, 1470, 1435, 1412, 1396, 1366, 1334, $1303\left(-\mathrm{SO}_{2}\right), 1278,1258,1233,1188,1174,1157\left(-\mathrm{SO}_{2}\right), 1135,1122,1083,1057,1031,1005,977$, 920, 849, 822, 805, 794, 786, 751, 705, 647, 620, 574, $555\left(-\mathrm{SO}_{2}\right), 523,480,459 . \mathrm{C}_{15} \mathrm{H}_{11} \mathrm{ClN}_{2} \mathrm{O}_{2} \mathrm{~S}$ (318.7): calcd. for: C:56.52, H: 3.48, Cl: 11.12, N: 8.79, O:10.04, S: 10.06, Found: C: 56.43, H: 3.56, $\mathrm{N}: 8.67, \mathrm{~S}: 10.10$. 
N-Quinoline-8-yl-benzenesulfonamide (5): Yield: $75 \%$, brown solid, Mp: 90-92 ${ }^{\circ} \mathrm{C},{ }^{1} \mathrm{H}-\mathrm{NMR}$ $\left(\mathrm{CDCl}_{3}, \delta \mathrm{ppm}\right): 7.32-7.43\left(\mathrm{~m}, 4 \mathrm{H},-H_{2-5}\right), 7.79\left(\mathrm{t}, 2 \mathrm{H}, J=8 \mathrm{~Hz},-H_{b}\right), 7.86\left(\mathrm{~d}, 2 \mathrm{H}, J=8 \mathrm{~Hz},-H_{a}\right), 7.88(\mathrm{t}$, $\left.1 \mathrm{H}, J=8 \mathrm{~Hz},-H_{c}\right), 8.07\left(\mathrm{~d}, 1 \mathrm{H}, J=8 \mathrm{~Hz},-H_{l}\right), 8.72\left(\mathrm{~d}, 1 \mathrm{H}, J=8 \mathrm{~Hz},-H_{6}\right), 11.21$ (br. $\left.1 \mathrm{H},-N H\right) .{ }^{13} \mathrm{C}-\mathrm{NMR}$ $\left(\mathrm{CDCl}_{3}, \mathrm{ppm}\right.$ ): 115.0 (Ar. -C), 122.0 (Ar. -C), 122.3 (Ar. -C), 125.9 (Ar. -C), 126.8 (Ar. -C), 127.2 (Ar. -C), 128.2 (Ar. -C), 128.9 (Ar. -C), 129.8 (Ar. -C), 132.9 (Ar. -C), 136.3 (Ar. -C), 139.3 (Ar. -C), 148.5 (Ar. -C). IR (cm $\left.{ }^{-1}\right): 3217$ (-NH), 2980, 2947, 2883, 1618, 1597, 1505, 1472, 1446, 1431, 1406, 1398, 1371, 1358, 1333, 1307 (-SO $), 1231,1169\left(-\mathrm{SO}_{2}\right), 1123,1086,1072,1057,1033,1016,997$, $952,926,852,820,805,786,750,726,685,666,611,584,568,557\left(-\mathrm{SO}_{2}\right), 518,462 . \mathrm{C}_{15} \mathrm{H}_{12} \mathrm{~N}_{2} \mathrm{O}_{2} \mathrm{~S}$ (284.2): calcd. for: C:63.36, H: 4.25, N: 9.85, O:11.25, S: 11.28, Found: C:63.47, H: 4.16, N: 9.78, O:11.34 S: 11.24 .

N-Quinoline-8-yl-4-(trifluoromethyl)-benzenesulfonamide (6): Yield: $71 \%$, brown solid, Mp: 128$130{ }^{\circ} \mathrm{C},{ }^{1} \mathrm{H}-\mathrm{NMR}\left(\mathrm{CDCl}_{3}, \delta \mathrm{ppm}\right): 7.42-7.63\left(\mathrm{~m}, 4 \mathrm{H},-H_{2-5}\right), 7.86\left(\mathrm{~d}, 2 \mathrm{H}, J=8 \mathrm{~Hz},-H_{b}\right), 8.03(\mathrm{~d}, 2 \mathrm{H}, J=8$ $\left.\mathrm{Hz},-H_{a}\right), 8.11\left(\mathrm{~d}, 1 \mathrm{H}, J=8 \mathrm{~Hz},-H_{l}\right), 8.75\left(\mathrm{~d}, 1 \mathrm{H}, J=8 \mathrm{~Hz},-H_{6}\right), 10.94$ (br. $\left.1 \mathrm{H},-N H\right) .{ }^{13} \mathrm{C}-\mathrm{NMR}\left(\mathrm{CDCl}_{3}\right.$,

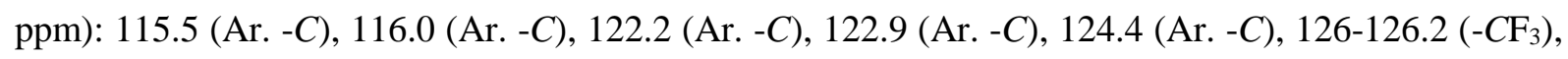
126.6 (Ar. -C), 126.8 (Ar. -C), 127.7 (Ar. -C), 128.3 (Ar. -C), 133.1 (Ar. -C), 136.4 (Ar. -C), 138.5 (Ar. -C), 148.9 (Ar. -C). IR (cm $\left.{ }^{-1}\right): 3239$ (-NH), 3106, 3069, 3051, 2980, 2947, 1603, 1581, 1505, 1473, 1435, 1403, 1371, 1321, 1307 (-SO $), 1255,1238,1163\left(-\mathrm{SO}_{2}\right), 1134,1104,1086,1061,1030,1007$, $972,922,830,795,759,711,656,634,603,591,575,551\left(-\mathrm{SO}_{2}\right), 521,492 . \mathrm{C}_{16} \mathrm{H}_{11} \mathrm{~F}_{3} \mathrm{~N}_{2} \mathrm{O}_{2} \mathrm{~S}(353.8)$ : calcd. for: C:54.54, H: 3.15, F: 16.18, N: 7.95, O:9.08, S: 9.10, Found: C:54.42, H: 3.21, N: 7.81, S: 9.13 .

General procedure for the synthesis of [(p-cymene)RuLCl], 7-12: A solution of 1-6 (0.50 mmol) in methyl alcohol $(5 \mathrm{ml})$ was added to a solution of $\left[\mathrm{RuCl}_{2}\right.$ (p-cymene $\left.)\right]_{2}(0.25 \mathrm{mmol})$ in methyl alcohol (5 $\mathrm{ml}$ ) in a Schlenk tube. The reaction mixture was stirred for 12 hours at $60^{\circ} \mathrm{C}$. The volatiles were removed under reduced pressure. The residue was washed with diethyl ether $(20 \mathrm{ml})$ and dried under vacuum. The desired products were recrystallized from $\mathrm{MeOH}$ to give orange or dark red-colored microcrystals (Figure 2).

\{[N-Quinoline-8-yl-4-t-butyl-benzenesulfonamido]-(p-cymene)-chloro-ruthenium(II)\} (7): Yield: $72 \%$, orange microcrystals, Mp: $291-292{ }^{\circ} \mathrm{C},{ }^{1} \mathrm{H}-\mathrm{NMR}\left(\mathrm{CDCl}_{3}, \delta \mathrm{ppm}\right): 0,95$ and 1,07 (d, d, $6 \mathrm{H}, J=8$ $\left.\mathrm{Hz},-H_{m}\right), 1.24\left(\mathrm{~s}, 9 \mathrm{H},-\mathrm{C}\left(\mathrm{CH}_{3}\right)_{3}\right), 2.37\left(\mathrm{~s}, 3 \mathrm{H},-H_{k}\right), 2.62\left(\mathrm{~m}, 1 \mathrm{H},-H_{l}\right), 5.70\left(\mathrm{~d}, 2 \mathrm{H}, J=4 \mathrm{~Hz},-H_{y}\right), 6.29$ (d, $\left.2 \mathrm{H}, J=4 \mathrm{~Hz},-H_{x}\right), 7.01-7.44\left(\mathrm{~m}, 4 \mathrm{H},-H_{2-5}\right), 8.07$ (d, $\left.1 \mathrm{H}, J=8 \mathrm{~Hz},-H_{l}\right), 8.15(\mathrm{dd}, 4 \mathrm{H}, J=4 \mathrm{~Hz}, J=4$ $\left.\mathrm{Hz},-\mathrm{H}_{a-b}\right), 9.10\left(\mathrm{~d}, 1 \mathrm{H}, J=8 \mathrm{~Hz},-\mathrm{H}_{6}\right) .{ }^{13} \mathrm{C}-\mathrm{NMR}\left(\mathrm{CDCl}_{3}, \mathrm{ppm}\right): 19.3\left(-\mathrm{CH}_{3}\right), 22.1\left(-\mathrm{CH}\left(\mathrm{CH}_{3}\right)_{2}\right), 30.9$ ($\left.\mathrm{CH}\left(\mathrm{CH}_{3}\right)_{2}\right), 31.1\left(-\mathrm{C}\left(\mathrm{CH}_{3}\right)_{3}\right), 34.8\left(-\mathrm{C}\left(\mathrm{CH}_{3}\right)_{3}\right), 82.3$ (Ar. -C), 84.6 (Ar. -C), 86.2 (Ar. - $\mathrm{C}$ ), 104.5 (Ar. C), 115.7 (Ar. -C), 118.0 (Ar. -C), 121.9 (Ar. -C), 125.4 (Ar. -C), 128.3 (Ar. -C), 129.0 (Ar. -C), 129.5 $($ Ar. $-C$ ), 137.8 (Ar. -C), 137.9 (Ar. -C), 144.7 (Ar. -C), 147.4 (Ar. -C), 151.1 (Ar. -C), 154.6 (Ar. -C). IR ( $\left.\mathrm{cm}^{-1}\right): 3055,2960,2906,2869,1639,1597,1570,1535,1530,1502,1462,1432,1376,1365,1315$ $\left(-\mathrm{SO}_{2}\right), 1298,1285,1273,1261,1212,1204,1190,1145\left(-\mathrm{SO}_{2}\right), 1107,1081,1057,1047,1015,947$, $905,877,868,822,803,787,749,735,712,690,665,649,623,577\left(-\mathrm{SO}_{2}\right), 549,523,470 . \mathrm{C}_{29} \mathrm{H}_{33} \mathrm{Cl}$ 
$\mathrm{N}_{2} \mathrm{O}_{2} \mathrm{RuS}$ (609/9): calcd. for: C:56,90, H:5,76, Cl:5,79, N:4,58, O:5,23, Ru:16,51, S:5,24, Found: C:56,80, H:5,92, N:4,57, S:5,23.

\{[N-Quinoline-8-yl-4-methoxy-benzenesulfonamido]-(p-cymene)-chloro-ruthenium(II)\}

(8):

Yield: $82 \%$, Dark red microcrystals, Mp: $194-196{ }^{\circ} \mathrm{C},{ }^{1} \mathrm{H}-\mathrm{NMR}\left(\mathrm{CDCl}_{3}, \delta \mathrm{ppm}\right): 0,92$ and 1,08 (d, d, $\left.6 \mathrm{H}, J=8 \mathrm{~Hz},-H_{m}\right), 2.36\left(\mathrm{~s}, 3 \mathrm{H},-H_{k}\right), 2.62\left(\mathrm{~m}, 1 \mathrm{H},-H_{l}\right), 3.72\left(\mathrm{~s}, 3 \mathrm{H},-\mathrm{OCH}_{3}\right), 5.67\left(\mathrm{~d}, 2 \mathrm{H}, J=4 \mathrm{~Hz},-H_{y}\right)$, $6.26\left(\mathrm{~d}, 2 \mathrm{H}, J=4 \mathrm{~Hz},-H_{x}\right), 6.77$ (d, $\left.2 \mathrm{H}, J=8 \mathrm{~Hz},-H_{b}\right), 7.55-7.77$ (m, 4H, $\left.-H_{2-5}\right), 8.06$ (d, $1 \mathrm{H}, J=8 \mathrm{~Hz},-$ $\left.H_{1}\right), 8.16\left(\mathrm{~d}, 2 \mathrm{H}, J=8 \mathrm{~Hz},-H_{a}\right), 9.10\left(\mathrm{~d}, 1 \mathrm{H}, J=8 \mathrm{~Hz},-H_{6}\right) .{ }^{13} \mathrm{C}-\mathrm{NMR}\left(\mathrm{CDCl}_{3}, \mathrm{ppm}\right): 19.3\left(-\mathrm{CH}_{3}\right), 22.1$ $\left(-\mathrm{CH}\left(\mathrm{CH}_{3}\right)_{2}\right), 31.0\left(-\mathrm{CH}\left(\mathrm{CH}_{3}\right)_{2}\right), 55.3\left(-\mathrm{OCH}_{3}\right), 80.6$ (Ar. -C), 82.3 (Ar. -C), 84.4 (Ar. -C), 86.2 (Ar. C), 113.5 (Ar. -C), 115.8 (Ar. -C), 117.9 (Ar. -C), 121.9 (Ar. -C), 128.9 (Ar. -C), 129.5 (Ar. -C), 130.6 (Ar. -C), 132.6 (Ar. -C), 137.8 (Ar. -C), 144.7 (Ar. -C), 147.4 (Ar. -C), 151.2 (Ar. -C), 161.8 (Ar. -C). IR ( $\left.\mathrm{cm}^{-1}\right)$ : 3091, 3047, 2962, 2904, 2875, 1642, 1592, 1568, 1534, 1498, 1466, 1440, 1412, 1387, 1373, $1316\left(-\mathrm{SO}_{2}\right), 1292,1285,1274,1252,1216,1191,1182,1157,1140\left(-\mathrm{SO}_{2}\right), 1134,1111,1082,1059$, $1045,1026,1018,1008,990,943,902,863,840,818,805,790,779,759,720,692,660,628,590,572$ (-SO $\mathrm{SO}_{2}$, 555, 527, 473. $\mathrm{C}_{26} \mathrm{H}_{27} \mathrm{Cl} \mathrm{N}_{2} \mathrm{O}_{3} \mathrm{RuS}$ (583.9): Calcd. for: C:53,28, H:4,99, Cl:6,05, N:4,78, O:8,19, Ru:17,24, S:5,47, Found: C:53,35, H:4,87, N:4,69, S:5,55.

\{[N-Quinoline-8-yl-4-n-propyl-benzenesulfonamido]-(p-cymene)-chloro-ruthenium(II)\}

(9): Yield: $76 \%$, dark red microcrystals, Mp: $247-248{ }^{\circ} \mathrm{C},{ }^{1} \mathrm{H}-\mathrm{NMR}\left(\mathrm{CDCl}_{3}, \delta \mathrm{ppm}\right): 0.83$ (t, $2 \mathrm{H}, J=8 \mathrm{~Hz}$, $\left.\mathrm{CH}_{2} \mathrm{CH}_{2} \mathrm{CH}_{3}\right), 0,92$ and 1,07 (d, d, 6H, $\left.J=8 \mathrm{~Hz},-\mathrm{H}_{m}\right), 1.37$ (t, 3H, J=8 Hz, $\left.-\mathrm{CH}_{2} \mathrm{CH}_{2} \mathrm{CH}_{3}\right), 2.34(\mathrm{~s}, 3 \mathrm{H}$, $\left.-H_{k}\right), 2.61\left(\mathrm{~m}, 1 \mathrm{H},-H_{l}\right), 3.10\left(\mathrm{~m}, 2 \mathrm{H},-\mathrm{CH}_{2} \mathrm{CH}_{2} \mathrm{CH}_{3}\right), 5.86\left(\mathrm{~d}, 2 \mathrm{H}, J=4 \mathrm{~Hz},-H_{y}\right), 6.24(\mathrm{~d}, 2 \mathrm{H}, J=4 \mathrm{~Hz},-$ $\left.H_{x}\right), 6.96-7.20\left(\mathrm{~m}, 4 \mathrm{H},-\mathrm{H}_{2-5}\right), 7.37\left(\mathrm{~d}, 2 \mathrm{H}, J=8 \mathrm{~Hz},-H_{b}\right), 8.04\left(\mathrm{~d}, 1 \mathrm{H}, J=8 \mathrm{~Hz},-H_{1}\right), 8.11(\mathrm{~d}, 2 \mathrm{H}, J=8 \mathrm{~Hz}$, $\left.-H_{a}\right), 9.10\left(\mathrm{~d}, 1 \mathrm{H}, J=8 \mathrm{~Hz},-\mathrm{H}_{6}\right) .{ }^{13} \mathrm{C}-\mathrm{NMR}\left(\mathrm{CDCl}_{3}, \mathrm{ppm}\right): 8.7\left(-\mathrm{CH}_{2} \mathrm{CH}_{2} \mathrm{CH}_{3}\right), 19.3\left(-\mathrm{CH}_{3}\right), 22.3(-$ $\left.\mathrm{CH}\left(\mathrm{CH}_{3}\right)_{2}\right), 24.1\left(-\mathrm{CH}_{2} \mathrm{CH}_{2} \mathrm{CH}_{3}\right), 30.9\left(-\mathrm{CH}\left(\mathrm{CH}_{3}\right)_{2}\right), 46.1\left(-\mathrm{CH}_{2} \mathrm{CH}_{2} \mathrm{CH}_{3}\right), 80.6$ (Ar. -C), 82.3 (Ar. -C), 84.5 (Ar. -C), 86.1 (Ar. -C), 115.8 (Ar. -C), 117.9 (Ar. -C), 121.9 (Ar. -C), 128.4 (Ar. -C), 128.5 (Ar. C), 128.9 (Ar. -C), 129.5 (Ar. -C), 137.8 (Ar. -C), 138.1 (Ar. -C), 144.7 (Ar. -C), 146.2 (Ar. -C), 147.4 (Ar. -C), 151.3 (Ar. -C). IR (cm $\left.{ }^{-1}\right)$ : 3049, 2977, 2953, 2926, 2868, 2851, 1595, 1569, 1531, 1505, 1464, 1423, 1398, 1378, 1363, $1316\left(-\mathrm{SO}_{2}\right), 1289,1269,1235,1214,1201,1189,1158,1136\left(-\mathrm{SO}_{2}\right), 1114$, 1081, 1044, 1019, 990, 947, 905, 872, 842, 825, 806, 793, 785, 761, 675, 652, 635, 605, 589, 577, 540, 528, 516, 505, 465. $\mathrm{C}_{28} \mathrm{H}_{31} \mathrm{ClN}_{2} \mathrm{O}_{2} \mathrm{RuS}$ (595.9): calcd. for: C:56,22, H:5,56, Cl:5,93 N:4,68, O:5,35, Ru:16,90, S:5,36, Found: C:56,31, H:5,48, N:4,60, S:5,40.

\{[N-Quinoline-8-yl-4-chloro-benzenesulfonamido]-(p-cymene)-chloro-ruthenium(II)\} (10): Yield: $83 \%$, dark red microcrystals, Mp: $171-172{ }^{\circ} \mathrm{C},{ }^{1} \mathrm{H}-\mathrm{NMR}\left(\mathrm{CDCl}_{3}, \delta \mathrm{ppm}\right): 0,94$ and 1,09 (d, d, $6 \mathrm{H}, J=8$ $\left.\mathrm{Hz},-H_{m}\right), 2.36\left(\mathrm{~s}, 3 \mathrm{H},-H_{k}\right), 2.63\left(\mathrm{~m}, 1 \mathrm{H},-H_{l}\right), 5.67\left(\mathrm{~d}, 2 \mathrm{H}, J=4 \mathrm{~Hz},-H_{y}\right), 6.23\left(\mathrm{~d}, 2 \mathrm{H}, J=4 \mathrm{~Hz},-H_{x}\right)$, 7.03-7.28 (m, 4H, $\left.-H_{2-5}\right), 7.36\left(\mathrm{~d}, 2 \mathrm{H}, J=8 \mathrm{~Hz},-H_{b}\right), 8.10$ (d, $\left.1 \mathrm{H}, J=8 \mathrm{~Hz},-H_{1}\right), 8.19$ (d, 2H, J=8 Hz, $\left.\mathrm{H}_{a}\right), 9.12\left(\mathrm{~d}, 1 \mathrm{H}, \mathrm{J}=8 \mathrm{~Hz},-\mathrm{H}_{6}\right) .{ }^{13} \mathrm{C}-\mathrm{NMR}\left(\mathrm{CDCl}_{3}, \mathrm{ppm}\right): 19.3\left(-\mathrm{CH}_{3}\right), 22.2-22.3\left(-\mathrm{CH}\left(\mathrm{CH}_{3}\right)_{2}\right), 31.0(-$ $\mathrm{CH}\left(\mathrm{CH}_{3}\right)_{2}$ ), 80.7 (Ar. -C), 82.4 (Ar. -C), 84.3 (Ar. -C), 85.9 (Ar. -C), 116.4 (Ar. -C), 117.9 (Ar. -C), 122.1 (Ar. -C), 127.7 (Ar. -C), 128.5 (Ar. -C), 128.9 (Ar. -C), 129.5 (Ar. -C), 130.2 (Ar. -C), 137.5 (Ar. -C), 137.9 (Ar. -C), 139.4 (Ar. -C), 144.7 (Ar. -C), 151.5 (Ar. -C). IR $\left(\mathrm{cm}^{-1}\right)$ : 3055, 3010, 2960, 2869, 
$1572,1532,1532,1502,1465,1446,1422,1392,1377,1315\left(-\mathrm{SO}_{2}\right), 1298,1278,1270,1233,1214$, 1189, 1173, 1161, $1140\left(-\mathrm{SO}_{2}\right), 1111,1080,1044,1031,1013,1005,985,965,946,905,868,833,821$, 805, 792, 781, 748, 708, 693, 666, 646, 614, 587, $567\left(-\mathrm{SO}_{2}\right), 541,524,501,480,470,461,455$. $\mathrm{C}_{25} \mathrm{H}_{24} \mathrm{Cl}_{2} \mathrm{~N}_{2} \mathrm{O}_{2} \mathrm{RuS}$ (588.4): calcd. for: C:50,85, H:4,44, Cl:12,01, N:4,74, O:5,42, Ru:17,12, S:5,43, Found: C:50,91, H:4,40, N:4,78, S:5,49.

\{[N-Quinoline-8-yl-benzenesulfonamido]-(p-cymene)-chloro-ruthenium(II)\} (11): Yield: $83 \%$, dark red microcrystals, Mp: 256-257 ${ }^{\circ} \mathrm{C},{ }^{1} \mathrm{H}-\mathrm{NMR}\left(\mathrm{CDCl}_{3}, \delta \mathrm{ppm}\right): 0,93$ and 1,08 (d, d, 6H, J=8 Hz, $\left.H_{m}\right), 2.37\left(\mathrm{~s}, 3 \mathrm{H},-H_{k}\right), 2.62\left(\mathrm{~m}, 1 \mathrm{H},-H_{l}\right), 5.69\left(\mathrm{~d}, 2 \mathrm{H}, J=4 \mathrm{~Hz},-H_{y}\right), 6.26\left(\mathrm{~d}, 2 \mathrm{H}, J=4 \mathrm{~Hz},-H_{x}\right), 6.98-$ $7.41\left(\mathrm{~m}, 4 \mathrm{H},-H_{2-5},-H_{b, c}\right), 8.06\left(\mathrm{~d}, 1 \mathrm{H}, J=8 \mathrm{~Hz},-H_{l}\right), 8.23\left(\mathrm{~d}, 2 \mathrm{H}, J=8 \mathrm{~Hz},-H_{a}\right), 9.11(\mathrm{~d}, 1 \mathrm{H}, J=8 \mathrm{~Hz},-$ $\left.\mathrm{H}_{6}\right) .{ }^{13} \mathrm{C}-\mathrm{NMR}\left(\mathrm{CDCl}_{3}, \mathrm{ppm}\right): 19.3\left(-\mathrm{CH}_{3}\right), 22.1,22.3\left(-\mathrm{CH}\left(\mathrm{CH}_{3}\right)_{2}\right), 30.9\left(-\mathrm{CH}\left(\mathrm{CH}_{3}\right)_{2}\right), 80.5$ (Ar. -C), 82.3 (Ar. -C), 84.4 (Ar. -C), 86.1 (Ar. -C), 116.0 (Ar. -C), 117.9 (Ar. -C), 121.9 (Ar. -C), 128.4 (Ar. C), 128.6 (Ar. -C), 128.9 (Ar. -C), 129.5 (Ar. -C), 131.3 (Ar. -C), 137.8 (Ar. -C), 140.9 (Ar. -C), 144.7 (Ar. -C), 147.2 (Ar. -C), 151.3 (Ar. -C). IR ( $\left.\mathrm{cm}^{-1}\right)$ : 3058, 2964, 2935, 2870, 1570, 1531, 1503, 1475 , 1464, 1445, 1423, 1378, $1316\left(-\mathrm{SO}_{2}\right), 1298,1290,1274,1234,1214,1189,1176,1165,1139\left(-\mathrm{SO}_{2}\right)$, 1112, 1087, 1044, 1028, 1010, 961, 946, 933, 898, 868, 850, 824, 817, 805, 795, 783, 777, 753, 714, 692, 662, 637, 614, 582 (-SO $\left.\mathrm{SO}_{2}\right), 565,539,524,502,473,463 . \mathrm{C}_{25} \mathrm{H}_{25} \mathrm{ClN}_{2} \mathrm{O}_{2} \mathrm{RuS}$ (553.9): calcd. for: C:54,00, H:4,89, Cl:6,38, N:5,04, O:5,75, Ru:18,18, S:5,77, Found: C: 54,12, H:4,81, N:5,14, S:5,69.

\section{\{[N-Quinoline-8-yl-4-(trifluoromethyl)-benzenesulfonamido]-(p-cymene)-chloro-ruthenium(II)\}}

(12): Yield: $83 \%$, dark red microcrystals, Mp: $245-247{ }^{\circ} \mathrm{C},{ }^{1} \mathrm{H}-\mathrm{NMR}\left(\mathrm{CDCl}_{3}, \delta \mathrm{ppm}\right): 0,94$ and 1,09 (d, $\left.\mathrm{d}, 6 \mathrm{H}, J=8 \mathrm{~Hz},-H_{m}\right), 2.36\left(\mathrm{~s}, 3 \mathrm{H},-H_{k}\right), 2.63\left(\mathrm{~m}, 1 \mathrm{H},-H_{l}\right), 5.68\left(\mathrm{~d}, 2 \mathrm{H}, J=4 \mathrm{~Hz},-H_{y}\right), 6.23(\mathrm{~d}, 2 \mathrm{H}, J=4$ $\left.\mathrm{Hz},-H_{x}\right), 7.05-7.41\left(\mathrm{~m}, 4 \mathrm{H},-H_{2-5}\right), 7.55$ (d, $\left.2 \mathrm{H}, J=8 \mathrm{~Hz},-H_{b}\right), 8.10$ (d, $\left.1 \mathrm{H}, J=8 \mathrm{~Hz},-H_{l}\right), 8.37$ (d, 2H, $\left.J=8 \mathrm{~Hz},-\mathrm{H}_{a}\right), 9.12\left(\mathrm{~d}, 1 \mathrm{H}, J=8 \mathrm{~Hz},-\mathrm{H}_{6}\right) .{ }^{13} \mathrm{C}-\mathrm{NMR}\left(\mathrm{CDCl}_{3}, \mathrm{ppm}\right): 19.3\left(-\mathrm{CH}_{3}\right), 22.1-22.3\left(-\mathrm{CH}\left(\mathrm{CH}_{3}\right)_{2}\right)$, $30.9\left(-\mathrm{CH}\left(\mathrm{CH}_{3}\right)_{2}\right), 80.7$ (Ar. -C), 82.4 (Ar. -C), 84.4 (Ar. -C), 85.9 (Ar. -C), 116.6 (Ar. -C), 117.9 (Ar. C), 122.2 (Ar. -C), 125.3-125.5 (-CF F $_{3}, 126.5$ (Ar. -C), 126.6 (Ar. -C), 128.9 (Ar. -C), 129.6 (Ar. -C), 133.0 (Ar. -C), 137.9 (Ar. -C), 138.7 (Ar. $-C$ ), 139.3 (Ar. $-C$ ), 144.6 (Ar. $-C$ ), 151.6 (Ar. $-C$ ). IR $\left(\mathrm{cm}^{-1}\right.$ ): 3035, 2967, 2929, 2872, 2812, 1607, 1573, 1538, 1505, 1468, 1402, 1379, 1317 (-SO $), 1294,1235$, 1215, 1186, 1160, $1144\left(-\mathrm{SO}_{2}\right), 1129,1107,1086,1061,1016,949,910,876,852,832,821,806,793$, 781, 770, 759, 736, 708, 666, 632, 609, 563 (- $\left.\mathrm{SO}_{2}\right), 541,524,504,472 . \mathrm{C}_{26} \mathrm{H}_{24} \mathrm{ClF}_{3} \mathrm{~N}_{2} \mathrm{O}_{2} \mathrm{RuS}$ (621.9). Calcd. for: C:50,20, H:3,89, Cl:5,70, F:9.16, N:4,50, O:5,14, Ru:16,25, S:5,15, Found: C:50,12, H:3,96, $\mathrm{N}: 4,40, \mathrm{~S}: 5,22$.

$\mathbf{X}$-ray structural analyses. Single crystal of each compound $\mathbf{8}$ and $\mathbf{9}$ was mounted under inert perfluoropolyether at the tip of glass fiber and cooled in the cryostream of a Bruker APEX2 CCD diffractometer. The structures were solved by direct methods (SIR97[53]) and refined by least-squares procedures on $F^{2}$ using SHELXL-97[54]. All $\mathrm{H}$ atoms attached to carbon were introduced in calculation in idealised positions and treated as riding models. The drawing of the molecules was realised with the help of ORTEP32[55]. Crystal data and refinement parameters are shown in Table 5. 
Table- 1. Crystal data for 8 and 9.

\begin{tabular}{|c|c|c|}
\hline Identification code & $\mathbf{8}$ & 9 \\
\hline Empirical formula & $\mathrm{C}_{26} \mathrm{H}_{27} \mathrm{Cl} \mathrm{N}_{2} \mathrm{O}_{3} \mathrm{Ru} \mathrm{S}$ & $\mathrm{C}_{28} \mathrm{H}_{31} \mathrm{Cl} \mathrm{N}_{2} \mathrm{O}_{2} \mathrm{Ru} \mathrm{S}$ \\
\hline Formula weight & 584.08 & 596.13 \\
\hline Temperature, $\mathrm{K}$ & $180(2)$ & $180(2)$ \\
\hline Wavelength, $\AA$ & 0.71073 & 0.71073 \\
\hline Crystal system & Monoclinic & Monoclinic \\
\hline Space group & $\mathrm{P} 2{ }_{1} / \mathrm{n}$ & $\mathrm{P} 2{ }_{1} / \mathrm{c}$ \\
\hline $\mathrm{a}, \AA$ & $14.4087(17)$ & $10.5377(12)$ \\
\hline $\mathrm{b}, \AA$ & $11.6057(14)$ & $13.6881(14)$ \\
\hline $\mathrm{c}, \AA$ & $16.2028(19)$ & $17.987(2)$ \\
\hline$\alpha\left(^{\circ}\right)$ & 90.0 & 90.0 \\
\hline$\beta\left(^{\circ}\right)$ & $116.146(2)$ & $95.771(5)$ \\
\hline$\gamma\left({ }^{\circ}\right)$ & 90.0 & 90.0 \\
\hline Volume, $\AA^{3}$ & $2432.2(5)$ & $2581.3(5)$ \\
\hline $\mathrm{Z}$ & 4 & 4 \\
\hline Density (calc), $\mathrm{Mg} / \mathrm{m}^{3}$ & 1.595 & 1.534 \\
\hline Abs. coefficient, $\mathrm{mm}^{-1}$ & 0.872 & 0.821 \\
\hline $\mathrm{F}(000)$ & 1192 & 1224 \\
\hline Crystal size, $\mathrm{mm}^{3}$ & $0.50 \times 0.45 \times 0.25$ & $0.48 \times 0.48 \times 0.10$ \\
\hline Theta range, ${ }^{\circ}$ & 2.25 to 30.03 & 1.87 to 29.24 \\
\hline Reflections collected & 49026 & 21457 \\
\hline Indpt reflections $\left(\mathrm{R}_{\text {int }}\right)$ & $7061(0.0242)$ & $6964(0.0243)$ \\
\hline Completeness, \% & 99.2 & 99.1 \\
\hline Absorption correction & Multi-scan & Multi-scan \\
\hline Max. and min. transmission & 0.7462 and 0.6219 & 0.7458 and 0.6462 \\
\hline Refinement method & $\mathrm{F}^{2}$ & $\mathrm{~F}^{2}$ \\
\hline Data /restraints/parameters & $7061 / 0 / 311$ & $6964 / 0$ / 320 \\
\hline Goodness-of-fit on $\mathrm{F}^{2}$ & 1.062 & 1.059 \\
\hline $\mathrm{R} 1, w R 2[\mathrm{I}>2 \sigma(\mathrm{I})]$ & $0.0292,0.0624$ & $0.0250,0.0581$ \\
\hline R1, wR2 (all data) & $0.0404,0.0706$ & $0.0298,0.0604$ \\
\hline Residual density, e. $\AA^{-3}$ & 1.066 and -0.586 & 0.477 and -0.496 \\
\hline CCDC deposition no. & 925980 & 925981 \\
\hline
\end{tabular}

\section{Acknowledgments}

We acknowledge the financial support granted by Erciyes University (ERUBAP), (FBY-12-3909, ID: 3909). 


\section{References}

[1] M. Tabatabaee, M. A.-Abbasnejad, N. Nozari, S. Sadegheian, and M. Ghasemzadeh, Acta Cryst. 2007, E63, o2099-o2100.

[2] G.E. Camí, M.E. Chacón Villalba, P. Colinas, G.A. Echeverría, G. Estiu, D.B. Soria, J. Mol. Struct. 2012, 1024, 110-116.

[3] J.B. Tommasino, G. Pilet, F. N.R. Renaud, G. Novitchi, V. Robert, D. Luneau, Polyhedron 2012, $37,27-34$.

[4] W. Wang, T. Zhang, F. Wang, M. Shi, Tetrahedron 2011, 67, 1523-1529.

[5] A. S. Culf, J. T. Gerig, P. G. Williams, J. Biomol. NMR 1997, 10, 293-299.

[6] (a) S. S. Krasnikova, I. K. Yakushchenko, S. N.Shamaev, Mol. Cryst. Liq. Cryst. 2007, 468, 439445, (b) J. Zhao, D. Zhao, H. Zhao, Huaxue Shiji 1997, 19, 257-260 (c) F. P. Dininno, R. N. Guthikonda, US Patent Publication, 1994, US 5294610 A 19940315.

[7] E. Kremer, G. Facchin, E. Estevez, P. Albores, E. J. Baran, E. J. Ellena, M. H. Torre, J. Inorg. Biochem. 2006, 100, 1167-1175.

[8] (a) I. Beloso, J. Castro, J. A. Garcia-Vazquez, P. Perez-Lourido, J. Romero, A. Sousa, Polyhedron 2003, 22, 1099-1111. (b) I. Beloso, J. Castro, J. A. Garcia-Vazquez, P. Perez-Lourido, J. Romero, A. Sousa, Eur. J. Inorg. Chem. 2004, 635-645. (c) I. Beloso, J. Castro, J. A. Garcia-Vazquez, P. Perez-Lourido, J. Romero, A. Sousa, Inorg. Chem. 2005, 44, 336-351. (d) I. Beloso, J. Castro, J. A. Garcia-Vazquez, P. Perez-Lourido, J. Romero, A. Sousa, Polyhedron 2006, 25, 2673-2682.

[9] A. Szadkowska, K. Zukowska, A. E. Pazio, K. Wozniak, R. Kadyrov, K. Grela, Organometallics 2011, 30, 1130-1138.

[10] W. Jin, X. Li, B. Wan, J. Org. Chem. 2011, 76, 484-491.

[11] N. A. Cortez, G. Aguirre, M. Parra-Hake, R. Somanathan, Tetrahedron Lett. 2009, 50, 2228-2231.

[12] B. Macíasa, I. García, M. V. Villa, J. Borrás, A. Castiñeiras, F. Sanz, -Polyhedron 2002, 21 12291234.

[13] B. Macíasa, I. García, M. V. Villa, J. Borrás, A. Castiñeiras, F. Sanz, Z. Anorg. Allg. Chem. 2003, 629, 255-260.

[14] L. E. da Silva, P. T. de S. Jr. A. C. Joussef, C. Piovezan, A. Neves, Quim. Nova 2008, 31, 11611164.

[15] H. Xu, L.F. Huang, L.M. Guo, Y.G. Zhang, X.M. Ren, Y. Song, J. Xie, J. Luminescence 2008, 128, $1665-1672$.

[16] M.Yan, T. Li, Z.Yang, Inorg. Chem.Commun. 2011, 14, 463-465.

[17] (a) S. Dayan, N. Özpozan Kalaycioğlu, Appl. Organomet. Chem. 2013, 27, 52-58. (b) N. Özdemir, S. Dayan, O. Dayan, M. Dinçer, N. Özpozan Kalaycıŏlu, Mol. Phys. 2012, DOI: 10.1080/00268976.2012. 742209. (c) S. Dayan, N. Özpozan Kalaycığlu, O. Dayan, N. Özdemir, M. Dinçer, O. Büyükgüngör, Dalton Trans. 2013, 42, 4957-4969. (d) S. Dayan, N. 
Kayaci, N. Ozpozan Kalaycioglu, O. Dayan, E. Ozturk, Inorg. Chim. Acta, (2013), DOI: 10.1016/j.ica.2013.03.004

[18] M. K. O’Neill, A. F. Trappey, P. Battle, C. L. Boswell, D. N. Blauch, Dalton Trans. 2009, 33913394.

[19] C. Deraeve, A. Maraval, L. Vendier, V. Faugeroux, M. Pitié, B. Meunier, Eur. J. Inorg. Chem. 2008, 5622-5631.

[20] M.K. Paira, J. Dinda, T.-H. Lu, A.R. Paital, C. Sinha, Polyhedron 2007, 26, 4131-4140.

[21] I. Yamada, R. Noyori, Org. Lett. 2000, 22, 3425.

[22] S.W. Seidel, T.J. Deming, Macromolecules 2003, 4, 96439.

[23] A.M. Hayes, D.J. Morris, G.J. Clarkson, M. Wills, J. Am. Chem. Soc. 2005, $20,7318$.

[24] J. Canivet, G. Labat, H. Stoeckli-Evans, G. Suss-Fink, Eur. J. Inorg. Chem. 2005, 22, 4493.

[25] T. Ohkuma, N. Utsumi, K. Tsutsumi, K. Murata, C. Sandoval, R. Noyori, J. Am. Chem. Soc. 2006, 278724.

[26] T. Ohkuma, K. Tsutsumi, N. Utsumi, N. Arai, R. Noyori, K. Murata, Org. Lett. 2007, 2, 255-257.

[27] J.E.D. Martins, D.J. Morris, B. Tripathi, M. Wills, J. Organomet. Chem. 2008, 23 3527-3532.

[28] A. Çetin, O. Dayan, Chinese J. Chem. 2009, 5, 978-982.

[29] J.E.D. Martins, G.J. Clarkson, M. Wills, Org. Lett. 2009, 4, 847.

[30] F.K. Cheung, A.J. Clarke, G.J. Clarkson, D.J. Fox, M.A. Graham, C. Lin, A.L. Criville, M. Wills, Dalton Trans. 2010, 5, 1395.

[31] O. Dayan, B. Çetinkaya, J. Mol. Cat. A.: Chem. 2007, 271, 134-141.

[32] O. Dayan, N. Özdemir, Z. Şerbetçi, M. Dinçer, B. Çetinkaya, O. Büyükgüngör, Inorg. Chim. Acta 2012, 392, 246-253.

[33] R. Noyori, S. Hashiguchi, Acc. Chem. Res. 1997, 30, 97.

[34] H. Turkmen, I Kani, B. Cetinkaya, Eur. J. Inorg. Chem. 2012, 28, 4494-4499.

[35] A. Bacchi, M. Balordi, R. Cammi, L. Elviri, C. Pelizzi, F. Picchioni, V. Verdolino, K. Goubitz, R. Peschar, P. Pelagatti, Eur. J. Inorg. Chem. 2008, 28, 4462-4473.

[36] M.M. Wei, M. Garcia-Melchor, J.C. Daran, C. Audin, A. Lledós, R. Poli, E. Deydier, E. Manoury, Organometallics 2012, 31, 6669-6680.

[37] D.Pandiarajan, R. Ramesh, J. Organomet. Chem. 2013, 723, 26-35.

[38] A. A. Mikhailine, R. H. Morris, Inorg. Chem. 2010, 49, 11039-11044.

[39] A. A. Mikhailine, M. I. Maishan, A. J. Lough, R. H. Morris, J. Am. Chem. Soc. 2012, 134, $12266-12280$.

[40] X. Guo, Y. Tang, X. Zhang, M. Lei, J. Phys. Chem. A 2011, 115, 12321-12330.

[41] D. E. Prokopchuk, J. F. Sonnenberg, N. Meyer, M. Z. Iuliis, A. J. Lough, R. H. Morris, Organometallics 2012, 31, 3056-3064.

[42] J. Dimroth, U. Schedler, J. Keilitz, R. Haag, R. Schomäcker, Adv. Synth. Catal. 2011, 353, 13351344. 
[43] R. Noyori, S. Hashiguchi, Acc. Chem. Res. 1997, 30, 2, 97.

[44] S. Günnaz, N. Özdemir, S. Dayan, O. Dayan, B. Çetinkaya, Organometallics 2011, 30, 4165-4173.

[45] N. Gurbuz, E.O. Ozcan, ,I. Ozdemir, B. Cetinkaya, O. Sahin, O. Buyukgungor, Dalton Trans. 2012, 41, 82330-2339.

[46] T. Marimuthu, H. B. Friedrich, ChemCatChem 2012, 4, 2090-2095.

[47] M. Aydemir, F. Durap, C. Kayan, A. Baysal, Y. Turgut, Synlett 2012, 19, 2777-2784.

[48] C. Aliende, M. Perez-Manrique, F.A. Jalon, B.R. Manzano, A.M. Rodriguez, G. Espino, Organometallics 2012, 31, 6106-6123.

[49] A. Zirakzadeh, R. Schuecker, N. Gorgas, K. Mereiter, F. Spindler, W. Weissensteiner, Organometallics 2012, 31, 4241-4250.

[50] J. Vaclavik, P. Kacer, M. Kuzma, L. Cerveny, Molecules 2011, 16, 5460-5495.

[51] S. Gulcemal, Appl. Organomet. Chem. 2012, 26, 246-251.

[52] S. Gulcemal, J.C. Daran, B. Cetinkaya, Inorg. Chim. Acta 2011, 365, 264-268.

[53] A. Altomare, M. Burla, M. Camalli, G. Cascarano, C. Giacovazzo, A. Guagliardi, A. Moliterni, G. Polidori, R. Spagna, R. J. Appl. Cryst. 1999, 32, 115-119.

[54] G. M. Sheldrick Acta Cryst. A 2008, 64, 112-122.

[55] L. J. Farrugia J. Appl. Cryst. 1997, 30, 565.

[56] D. Carmona, M.P. Lamata, F.Viguri, R. Rodriguez, F.J. Lahoz, I.T. Dobrinovitch, L. A. Oro, Dalton Trans. 2008,3328.

[57] A.Singh, N.Singh, D.S.Pandey J.Organomet. Chem. 2002, 642, 48

[58] B.Therrien, G.Suss-Fink, P.Govindaswamy, C.Said-Mohamed Polyhedron 2007, 26, 4065.

[59] A. Bacchi, P. Pelagatti, C. Pelizzi, D. Rogolino, J. Organomet. Chem. 2009, 694, 3200-3211.

[60] C. A. Madrigal, A. García-Fernández, J. Gimeno, E. Lastra, J. Organomet. Chem. 2008, 693, 25352540.

[61] P. Kumar, A. K. Singh, R. Pandey, P. Z. Li, S. K. Singh, Q. Xu, D. S. Pandey, J. Organomet. Chem. 2010, 695, 2205-2212.

[62] M. Aydemir, N. Meric, A. Baysal, Y. Turgut, C. Kayan, S. Seker, M. Toğrul, B. Gümgüm, J. Organomet. Chem. 2011, 696, 1541-1546

[63] D. Elma, F. Durap, M. Aydemir, A. Baysal, N. Meric, B. Ak, Y. Turgut, B. Gümgüm, J. Organomet. Chem. 2013, 729, 46-52.

[64] B. T. Kurkar, H. Turkmen, I. Kani, B. Cetinkaya, Tetrahedron 2012, 68, 8655-8662

[65] N. A. Cortez, G. Aguirre, M. Parra-Hake, R. Somanathan, Tetrahedron Asymm. 2008, 19, 13041309.

[66] J. E. D. Martins, M. A. C. Redondo, M. Wills, Tetrahedron: Asymm. 2010, 21, 2258-2264

[67] A. Grabulosa, A. Mannu, E. Alberico, S. Denurra, S. Gladiali, G. Muller, J. Mol. Catal. A Chem. 2012, 363, 49- 57.

[68] M. U. Raja, E. Sindhuja, R. Ramesh, Inorg. Chem. Commun. 2010, 13, 1321-1324. 
[69] M. C. Carrión, F. A. Jalón, B. R. Manzano, A. M. Rodríguez, F. Sepúlveda, M. Maestro Eur. J. Inorg. Chem. 2007, 3961-3973.

[70] T. Glöge, D. Petrovic, C. Hrib, P. G. Jones, M. Tamm Eur. J. Inorg. Chem. 2009, 4538-4546

[71] D. J. Morris, A. M. Hayes, M. Wills J. Org. Chem. 2006, 71, 7035-7044.

[72] P. Singh, A. K. Singh, Organometallics, 2010, 29, 6433-6442.

[73] M. Ito, Y. Shibata, A. Watanabe, T. Ikariya, Synlett, 2009, 10, 1621-1626. 